tracted from it with benzene. The mother liquors, containing a small amount of psoralen, were extracted with chloroform. The concentrated extract was passed through activated alumina (Brockmann activity II-III) and the psoralen was eluted with benzene. The content of psoralen in the technical product was determined by the polarographic method [4].

It can be seen from the table that the most desirable raw material for the industrial production of psoralen is formed by the roots and seeds. However, the seeds are preferable since they contain a larger amount of psoralen and, in addition, the natural thickets of the plants are preserved. The best period for collecting the seeds is from the second half of July to the end of August.

\title{
REEERENCES
}

1. T. T. Shakirov, I. N. Kartashkina, and M. R. Shamsutdinov, Khim. farm. zh., no. 4, 52, 1967; Author's certificate no. 200736, Byull. izobr. no. 17, 1967.

2. N. K. Abubakirov and U. F. Khalmurzaev, KhPS [Chemistry of Natural Compounds], 3, $137,1967$.

3. Flora USSR, Vol. IX [in Russian]. Moscow and Leningrad, p. 300, 1945.

4. Yu. E. Orlov and N. P. Dzyuba, Med. prom. SSSR, no. 4, 44, 1965.

4 January 1968

Institute of the Chemistry of Plant Substances AS UzSSR

UDC 661.728 .31

\section{A STUDY OF THE ANTHOCYANINS OF THE COTTON PLANT}

Z. B. Rakhimkhanov, A. K. Karimdzhanov, A. I. Ismailov, and A. S. Sadykov

Khimiya Prirodnykh Soedinenii, Vol. 4, No. 3, pp. 190-191, 1968

We have extracted flowers of cotton plants of variety $315-\mathrm{F}$ (Gossypium hirsutum) with methanol in $1 \%$ hydrochloric acid. The anthocyanins were precipitated from the extract with ether; they were freed from contaminating flavonols, catechins, and waxes by chromatography on a column of cellulose powder. As eluant we used system 1:1butanol-acetic acid-water $(4: 1: 5)$.

On paper chromatography in system 2 [water-acetic acid-concentrated hydrochloric acid (82:15:3)], two anthocyaninins were detected with $R_{f} 0.25$ (I) and 0.36 (II). The anthocyanins were separated by chromatography on a column of cellulose powder with the same solvent (II). The clearly separated zones were cut out and eluted with methanol containing $0.01 \%$ of hydrochloric acid. After recrystallization from ethanol $2 \mathrm{~N}$ with respect to hydrochloric acid, anthocyanin (I) was isolated with mp $215-217^{\circ} \mathrm{C}$ (decomp) and composition $\mathrm{C}_{21} \mathrm{H}_{21} \mathrm{O}_{11} \mathrm{Cl} \cdot 2 \mathrm{H} \mathrm{H}_{2} \mathrm{O}$. The paper chromatography of anthocyanin (I) in the presence of chrysanthemin (from the aster) in system 2 showed that they were identica1.

The hydrolysis of the anthocyanin with $2 \mathrm{~N}$ hydrochloric acid led to the decomposition of the substance into the aglycone and glucose. The glucose was identified by paper chromatography in two systems: ethyl acetate-pyridinewater $(2: 1: 2)$ and 1 -butanol-acetic acid-water $(4: 1: 5)$. The position of the OH group in the anthocyanin was shown by its absorption spectra. The UV spectrum of the glycoside had its maximum absorption at $525 \mathrm{~m} \mu$ or, in the presence of aluminum chloride, at $568 \mathrm{~m} \mu[1,2]$. The anthocyanidin cyanidin was identified by comparing it with the cyanidin obtained from red rose petals (on chromatograms) and by absorption spectra (maxima at $535 \mathrm{~m} \mu$ ).

When the anthocyanidin was heated in $15 \%$ barium hydroxide for 30 min at $100^{\circ} \mathrm{C}$, phloroglucinol and protocatechuic acid were formed, these being identified by paper chromatography in various systems in the presence of authentic samples of phloroglucinol and protocatechuic acid.

The results obtained show that the anthocyanin ( 1 ) from the cotton plant is chrysanthemin (cyanidin 3 - $\beta$-glucoside).

\section{REEERENCES}

1. C. O. Chichester and B. S. Lu, J. Food Sci., 30, 5-12, 1965.

2. I. B. Harborne, Biochem. J., 70, 22, 1958.

12 January 1968

Scientific Research Institute for the Chemistry and Technology of Cotton Cellulose, Tashkent 